Pacific Journal of Mathematics

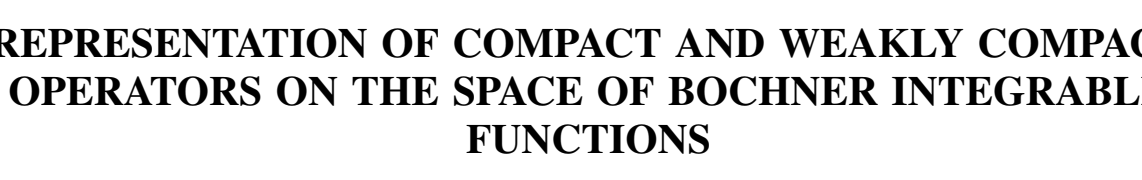




\title{
REPRESENTATION OF COMPACT AND WEAKLY COMPACT OPERATORS ON THE SPACE OF BOCHNER INTEGRABLE FUNCTIONS
}

\author{
KeVIN T. ANDREWS
}

If $X^{*}$ has the Radon-Nikodym property, then for every compact operator $T: L_{1}(\mu, X) \rightarrow Y$ there is a bounded function $g: \Omega \rightarrow L(X, Y)$ that is measurable for the uniform operator topology on $L(X, Y)$ such that

$$
T(f)=\int_{\Omega} f g d \mu
$$

for all $f$ in $L_{1}(\mu, X)$. The same result holds for weakly compact operators if $X^{*}$ is separable Schur space. These representations yield Radon-Nikodym theorems for operator valued measures and a generalization of a theorem of $D . R$. Lewis.

The representation of linear operators on the Banach space $L_{1}(\mu, X)$ of Bochner integrable functions, has been the object of much study for the past forty years. Dunford and Pettis began this investigation in 1940 [6] with the representation of weakly compact and norm compact operators on $L_{1}(\mu)$ by a Bochner integral. Their work was based on an earlier paper of Pettis [9] and was complemented by the work of Phillips [11]. More recently, the theory of liftings has been used by Dinculeanu [5] and others to obtain a representation for the general linear operator on $L_{1}(\mu, X)$. In this paper we will use methods in the spirit of Dunford, Pettis, and Phillips to show that if $X^{*}$ has the Radon-Nikodym property, then the compact operators on $L_{1}(\mu, X)$ are representable by measurable kernels and if $X^{*}$ is a separable Schur space (i.e., weakly convergent sequences converge in norm) then the weakly compact operators on $L_{1}(\mu, X)$ are representable by measurable kernels. As corollaries, we obtain a Radon-Nikodym theorem for operator-valued measures and a generalization of a theorem of D. R. Lewis [4, p. 88] on weakly measurable functions that are equivalent to norm measurable functions.

Throughout this paper $(\Omega, \Sigma, \mu)$ is a finite measure space and $X, Y$ and $Z$ are Banach spaces with duals $X^{*}, Y^{*}$, and $Z^{*}$ respectively. The space of all bounded linear operators from $X$ to $Y$ will be denoted by $L(X, Y)$. The subspaces of $L(X, Y)$ consisting of all the weakly compact and norm compact operators from $X$ to $Y$ will be denoted by $W(X, Y)$ and $K(X, Y)$. The space $L_{1}(\mu, X)$ is the space of $\mu$-Bochner integrable functions on $\Omega$ with values in $X$ and 
$L_{\infty}(\mu, X)$ is the space of $X$-valued $\mu$-Bochner integrable functions on $\Omega$ that are essentially bounded. An operator $T: L_{1}(\mu, X) \rightarrow Y$ is representable by a measurable kernel if there is a bounded measurable $g: \Omega \rightarrow L(X, Y)$ such that

$$
T(f)=\text { Bochner }-\int_{\Omega} f g d \mu .
$$

From this, it follows shat $\|T\|=\|g\|_{\infty}[5$, p. 283]. Recall that a Banach space is weakly compactly generated if it is the closed linear span of one of its weakly compact sets. Finally, note that if $\pi$ is a partition of $\Omega$ into a countable number of disjoint elements of $\Sigma$ and if $f$ is in $L_{1}(\mu, X)$, then the function $E_{\pi}: L_{1}(\mu, X) \rightarrow L_{1}(\mu, X)$ defined by

$$
E_{\pi}(f)=\sum_{E \in \pi} \frac{\int_{E} f d \mu}{\mu E} \chi_{E}
$$

(here the convention $0 / 0=0$ is observed) is a linear operator.

Most of the first lemma is well-known so we omit the proof.

LEMMA 1. For each countable partition $\pi$, the operator $E_{\pi}$ is a contraction on $L_{1}(\mu, X)$ and $L_{\infty}(\mu, X)$. Moreover, if the partitions are directed by refinement, then

$$
\begin{array}{llll}
\lim _{\pi}\left\|E_{\pi}(f)-f\right\|_{1}=0 & \text { for all } f \text { in } & L_{1}(\mu, X) \\
\lim _{\pi}\left\|E_{\pi}(f)-f\right\|_{\infty}=0 & \text { for all } f \text { in } & L_{\infty}(\mu, X) .
\end{array}
$$

Before stating the main theorem we require a preliminary definition. A function $g$ in $L_{\infty}(\mu, L(X, Y))$ is said to have its essential range in the uniformly (weakly) compact operators if there is a (weakly) compact set $C$ in $Y$ such that $g(\omega) x \in C$ for almost all $\omega$ in $\Omega$ and $x$ in $X$ with $\|x\| \leqq 1$.

THEOREM 2. Let $X^{*}$ have the Radon-Nikodym property. Then there is an isometric isomorphism between the space of compact operators $K\left(L_{1}(\mu, X), Y\right)$ and the subpace of $L_{\infty}(\mu, K(X, Y))$ consisting of theose functions whose essential range is in the uniformly compact operators. In fact, $T$ in $K\left(L_{1}(\mu, X), Y\right)$ and $g$ in $L_{\infty}(\mu, K(X, Y))$ are in correspondence if and only if

$$
T(f)=\int_{\Omega} \text { fgd } \mu \quad \text { for all } f \text { in } L_{1}(\mu, X) .
$$

Proof. Let $T$ be in $K\left(L_{1}(\mu, X), Y\right)$. Notice that for any par- 
tition $\pi, f$ in $L_{1}(\mu, X)$, and $g$ in $L_{\infty}\left(\mu, X^{*}\right)=\left(L_{1}(\mu, X)\right)^{*}$, we have that

$$
\int_{\Omega} E_{\pi}(f) g d \mu=\int_{\Omega} f E_{\pi}(g) d \mu .
$$

It follows from this that the adjoint of $T E_{\pi}$ is $E_{\pi} T^{*}$. Now, if the partitions $\pi$ are countable, we have that

$$
\lim _{\pi} E_{\pi} f=f \quad \text { for all } f \text { in } L_{\infty}\left(\mu, X^{*}\right)
$$

by Lemma 1 . Since $\left\|E_{\pi}\right\|_{\infty} \leqq 1$, this limit is uniform on compact sets. By Schauder's theorem, $T^{*}: Y^{*} \rightarrow L_{\infty}\left(\mu, X^{*}\right)$ is compact and so

$$
\lim _{\pi} E_{\pi} T^{*} y^{*}=T y^{*}
$$

uniformly for $\left\|y^{*}\right\| \leqq 1$. Therefore,

$$
\lim _{\pi} E_{\pi} T^{*}=T^{*}
$$

in the operator norm. Since $E_{\pi} T^{*}=\left(T E_{\pi}\right)^{*}$, it follows that

$$
\lim _{\pi} T E_{\pi}=T
$$

in operator norm.

Now, for each countable partition $\pi$, define $g_{\pi}: \Omega \rightarrow L(X, Y)$ by

$$
g_{\pi}(\cdot) x=\sum_{A \in \pi} \frac{T\left(x \chi_{A}\right)}{\mu A} \chi_{A}(\cdot) \text {. }
$$

Then for each partition $\pi, \omega$ in $\Omega$, and $x$ in $X$ with $\|x\| \leqq 1$, we have that $g_{\pi}(\omega) x \leqq T\left\{f: f\right.$ in $\left.L_{1}(\mu, X),\|f\|_{1} \leqq 1\right\}$. Since $T$ is compact, it follows that $g_{\pi}(\omega)$ is in $K(X, Y)$ for each partition $\pi$ and $\omega$ in $\Omega$. Moreover, one easily sees that

$$
T E_{\pi}(f)=\int_{\Omega} f g_{\pi} d \mu
$$

for all simple functions $f$ in $L_{1}(\mu, X)$ and thus for all functions $f$ in $L_{1}(\mu, X)$. Hence if $\pi_{1}$ and $\pi_{2}$ are two partitions, then

$$
\left(T E_{\pi_{1}}-T E_{\pi_{2}}\right)(f)=\int_{\Omega} f\left(g_{\pi_{1}}-g_{\pi_{2}}\right) d \mu .
$$

Since

$$
\lim _{\pi_{1}, \pi_{2}}\left\|T E_{\pi_{1}}-T E_{\pi_{2}}\right\|=0,
$$

an appeal to $[5$, p. 283] establishes that 


$$
\lim _{\pi_{1}, \pi_{2}}\left\|g_{\pi_{1}}-g_{\pi_{2}}\right\|_{\infty}=\lim _{\pi_{1}, \pi_{2}}\left\|T E_{\pi_{1}}-T E_{\pi_{2}}\right\|=0
$$

Thus the net $\left(g_{\pi}\right)$ is Cauchy in the norm of $L_{\infty}(\mu, K(X, Y))$. It follows that there is a $g$ in $L_{\infty}(\mu, K(X, Y))$ such that

$$
\lim _{\pi}\left\|g_{\pi}-g\right\|_{\infty}=0
$$

and so

$$
\lim _{\pi} \int_{\Omega} f g_{\pi} d \mu=\int_{\Omega} f g d \mu
$$

for all $f$ in $L_{1}(\mu, X)$. We also have, for almost all $\omega$, that

$$
\left.g(\omega) x \leqq \overline{T\left\{f: f \in L_{1}(\mu, X),\|f\| \leqq 1\right.}\right\}
$$

for all $x$ in $X$ with $\|x\| \leqq 1$. Hence the essential range of $g$ consists of uniformly compact operators. Finally, Lemma 1 ensures that

$$
T(f)=\lim _{\pi} T E_{\pi}(f)=\lim _{\pi} \int_{\Omega} f g_{\pi} d \mu=\int_{\Omega} f g d \mu .
$$

Conversely, suppose that $g: \Omega \rightarrow K(X, Y)$ is a bounded measurable function such that there is a compact set $C \subset Y$ with $g(\omega) x$ in $C$ for almost all $\omega$ in $\Omega$ and all $x$ in $X$ with $\|x\| \leqq 1$. Without loss of generality, we may assume $g(\omega) x$ is in $C$ for all $\omega$ in $\Omega$. Define

$$
T(f)=\int_{\Omega} f g d \mu
$$

for $f \in L_{1}(\mu, X)$. Another appeal to [5, p. 283] shows $\|T\|=\|g\|_{\infty}$. Let

$$
f=\sum_{i=1}^{n} x_{i} \chi_{E_{i}}
$$

be a simple function in $L_{1}(\mu, X)$ with $\|f\| \leqq 1$ i.e.,

$$
\sum_{i=1}^{n}\left\|x_{i}\right\| \mu E_{i} \leqq 1
$$

Then

$$
\begin{aligned}
T(f) & =\int_{\Omega} g f d \mu=\sum_{i=1}^{n} \int_{E_{i}} g(\omega) x_{i} d \mu(\omega) \\
& =\sum_{i=1}^{n}\left\|x_{i}\right\| \mu E_{i} \cdot \frac{1}{\mu E_{i}} \int_{E_{i}} g(\omega) \frac{x_{i}}{\left\|x_{i}\right\|} d \mu
\end{aligned}
$$

is in $\overline{c o} C$ by $[4$, p. 48]. Since $\overline{\text { co }} C$ is compact by Mazur's theorem, the operator $T$ is compact. This completes the proof. 
That $X^{*}$ has the Radon-Nikodym property is necessary as well as sufficient for the first part of the above proof. Indeed, if each $T$ in $K\left(L_{1}(\mu, X), Y\right)$ is representable by a Bochner integrable $g$ in $L_{\infty}(\mu, K(X, Y))$, then taking $Y$ to be the scalars shows that $L_{1}(\mu, X)^{*}=$ $L_{\infty}\left(\mu, X^{*}\right)$ which implies $\left[4\right.$, p. 98] that $X^{*}$ has the RNP. An immediate consequence of Theorem 2 is a Radon-Nikodym theorem for certain operator valued measures.

Corollary 3. Let $X^{*}$ have the RNP and let $G: \Sigma \rightarrow K(X, Y)$ be a $\mu$-continuous vector measure of bounded variation. If, for each $E_{1}$ in $\Sigma$ with $\mu E_{1}>0$, there exists $E_{2}$ in $\Sigma$ with $E_{2} \subseteq E_{1}$ and $\mu\left(E_{2}\right)>0$ such that

$$
\left\{\frac{G(E) x}{\mu(E)}: x \in X, E \in \Sigma, E \subseteq E_{2}, \mu(E)>0,\|x\| \leqq 1\right\}
$$

is relatively norm compact, then there exists a Bochner integrable $g: \Omega \rightarrow K(X, Y)$ such that

$$
G(E)=\int_{E} g d \mu
$$

for each $E$ in $\Sigma$.

Proof. By exhaustion [4, p. 70], the corollary is established if for each $E_{1}$ in $\Sigma$ with $\mu\left(E_{1}\right)>0$ we can find $E_{2}$ in $\Sigma$ with $E_{2} \subseteq E_{1}$ and $\varkappa_{2}>0$ and a Bochner integrable $g$ such that

$$
G(E)=\int_{E} g d \mu
$$

for all $E$ in $\Sigma$ with $E \subseteq E_{2}$. So let $E_{1} \in \Sigma$ with $\mu\left(E_{1}\right)>0$ and select the $E_{2} \subseteq E_{1}$ guaranteed by the hypothesis. Define an operator $T$ on the simple functions in $L_{1}(\mu, X)$ by

$$
T(f)=\sum_{i=1}^{n} G\left(A_{1} \cap E_{2}\right) x_{\imath} \quad \text { if } \quad f=\sum_{i=1}^{n} x_{i} \chi_{A_{i}}, A_{i} \quad \text { in } \quad \Sigma, A_{i} \cap A_{j}=\dot{\phi}
$$

if $i \neq j$. Notice that if $\|f\| \leqq 1$

$$
\sum_{i=1}^{n}\left\|x_{i}\right\| \mu A_{i} \leqq 1,
$$

then

$$
\sum_{i=1}^{n}\left\|x_{i}\right\| \mu\left(A_{i} \cap E_{2}\right) \leqq 1
$$

and so 


$$
T(f)=\sum_{i=1}^{n}\left\|x_{i}\right\| \mu\left(A_{i} \cap E_{2}\right) \cdot \frac{G\left(A_{i} \cap E_{2}\right) \frac{x_{i}}{\left\|x_{i}\right\|}}{\mu\left(A_{i} \cap E_{2}\right)}
$$

is in

$$
\overline{\mathrm{co}}\left\{\frac{G(E) x}{\mu E}: x \in X, E \in \Sigma, E \subseteq E_{2}, \mu(E)>0,\|x\| \leqq 1\right\},
$$

a set which is compact by Mazur's theorem. Thus $T$ has a compact linear extension to all of $L_{1}(\mu, X)$. Hence, by Theorem 2, there exists a Bochner integrable $g: \Omega \rightarrow K(X, Y)$ such that

$$
T(f)=\int_{\Omega} f g d \mu
$$

for all $f \in L_{1}(\mu, X)$. In particular, if $E$ is in $\Sigma$ and $E \subseteq E_{2}$, then

$$
G(E) x=T\left(x \chi_{E}\right)=\int_{E} g x d \mu .
$$

Since $g$ is Bochner integrable, we have, by [4, p. 47], that

$$
G(E)=\int_{E} g d \mu
$$

as required.

Our next result is a generalization of a theorem of D. R. Lewis $[4$, p. 88$]$ dealing with the equivalence of weakly measurable and measurable functions. The proof uses the following result of Amir and Lindenstrauss [1, p. 43]: If $X$ is a weakly compactly generated space and $X_{0} \subseteq X$ and $Y_{0} \subseteq X^{*}$ are separable subspaces, then there is a bounded projection $P: X \rightarrow X$ with separable range such that $X_{0} \subseteq P(X)$ and $Y_{0} \subseteq P^{*}\left(X^{*}\right)$.

Proposition 4. Let $X^{*}$ and $Y$ be weakly compactly generated Banach spaces. If $f: \Omega \rightarrow K(X, Y)$ is a bounded function such that for each $y^{*}$ in $Y^{*}$ the function $y^{*} f(\cdot): \Omega \rightarrow X^{*}$ is measurable, then there is a bounded measurable function $g: \Omega \rightarrow K(X, Y)$ such that for each $y^{*}$ in $Y^{*}, y^{*} f(\cdot)=y^{*} g(\cdot) \mu$-a.e., (the exceptional set may depend on $\left.y^{*}\right)$.

Proof. We claim that the set $A=\left\{y^{*} f(\cdot): y^{*} \in Y^{*},\left\|y^{*}\right\| \leqq 1\right\}$ is compact in $L_{1}\left(\mu, X^{*}\right)$. If not, then there is a sequence $y_{n}^{*}$ in the unit ball of $Y^{*}$ and $\delta>0$ such that

$$
\left\|y_{n}^{*} f(\cdot)-y_{m}^{*} f(\cdot)\right\|_{L_{1}\left(\mu, X^{*}\right)}>\delta
$$

for $m \neq n$. Choose a bounded projection $P_{1}: Y \rightarrow Y$ with separable 
range such that $P_{1}^{*} y_{n}^{*}=y_{n}^{*}$ for all $n$. Since each $y_{n}^{*} f(\cdot): \Omega \rightarrow X^{*}$ is measurable and hence essentially separably valued, there is a bounded projection $P_{2}: X^{*} \rightarrow X^{*}$ with separable range and sets $\Omega_{n}$ in $\Sigma$ with $\mu\left(\Omega \backslash \Omega_{n}\right)=0$ and $y_{n}^{*} f\left(\Omega_{n}\right) \subseteq P_{2}\left(X^{*}\right)$ for every $n$. Now, since each $f(\omega)$ is a compact operator we have, for all $x^{* *}$ in $X^{* *}$, that $f(\omega)^{* *} x^{* *}$ is in the natural image of $Y$ in $Y^{* *}$ and so we may define $h: \Omega \rightarrow$ $K\left(X^{* *}, Y\right)$ by $h(\omega) x^{* *}=P_{1} f(\omega)^{* *} P_{2}^{*} x^{* *}$. We claim that for each $x^{* *}$ in $X^{* *}$, the function $h(\cdot) x^{* *}: \Omega \rightarrow Y$ is measurable. To see this, note that since $P_{1}$ has separable range, the functions $h(\cdot) x^{* *}$ are separably valued and since

$$
y^{*} h(\cdot) x^{* *}=y^{*} P_{1} f(\cdot)^{* *} P_{2}^{*} x^{* *}=x^{* *} P_{2} f(\cdot)^{*} P_{1}^{*} y^{*}
$$

and each $f(\cdot) P_{1} y^{*}: \Omega \rightarrow X^{*}$ is measurable, the functions $h(\cdot) x^{* *}$ are weakly measurable. An appeal to the Pettis measurability theorem $\left[4\right.$, p. 42] establishes the measurability of $h(\cdot) x^{* *}$. Now if $Y_{0}$ is the Banach space obtained by taking the closed linear span of $P_{1} Y$ in $Y$, then $Y_{0}$ is separable and $h$ can be viewed as taking its values in $K\left(X^{* *}, Y_{0}\right)$. Moreover, if we define $S: Y \rightarrow Y_{0}$ by $S y=P_{1} y$, then $h(\omega) x^{* *}=S P_{1} f(\omega)^{* *} P_{2}^{*} x^{* *}$. Thus, if $y_{0}^{*}$ is in $Y_{0}^{*}$, then $h(\omega)^{*} y_{0}^{*}=$ $P_{2}^{* *} f(\omega)^{* *} P_{1}^{*} S^{*} y_{0}^{*}$ is in $P_{2} X^{*}$, since the range of $f(\omega)^{* * *}$ is in $X^{*}$ and $P_{2}^{* *}$ extends $P_{2}$. Let $Z=\widetilde{P_{2} X^{*}}$ and $B=\left\{T: T\right.$ in $K\left(X^{* *}, Y_{0}\right)$, $T^{*} Y_{0}^{*} \subset Z$ \}. We claim that $B$ is separable. To see this, let $U$ and $V$ denote the closed unit balls of $Z^{*}$ and $Y_{0}^{*}$ endowed with the weak* topologies. Since $Y_{0}$ and $Z$ are separable, $U$ and $V$ are compact metric spaces, and thus, so is $U \times V$. For each $T$ in $B$, define a function $J T$ on $U \times V$ by $J T(u, v)=u T^{*} v$. Then the map $T \rightarrow J T$ is a linear isometry of $B$ into $C(U \times V)$ [8] and so, by [7, p. 437], $B$ is separable. Since the values of $h$ in $K\left(X^{* * *}, Y_{0}\right)$ lie in $B$ and $\left\|h\left(\omega_{1}\right)-h\left(\omega_{2}\right)\right\|_{K\left(X^{* *}, Y\right)}=$ $\left\|h\left(\omega_{1}\right)-h\left(\omega_{2}\right)\right\|_{K\left(X^{* *}, Y_{0}\right)}$ for all $\omega_{1}, \omega_{2}$ in $\Omega$, the values of $h$ in $K\left(X^{* *}, Y\right)$ form a separable set. Now because $h(\cdot) x^{* *}$ is measurable for each $x^{* *}$ in $X^{* *}$, an appeal to $[5$, p. 102] establishes that $h$ is measurable. Since $h$ is bounded, $h$ is Bochner integrable and so we may choose a sequence $h_{n}$ of $K\left(X^{* *}, Y\right)$-valued simple functions such that

$$
\lim _{n} \int_{\Omega}\left\|h-h_{n}\right\| d \mu=0 .
$$

Define operators $S_{n}$ and $S$ from $L_{\infty}\left(\mu, X^{* *}\right)$ to $Y$ by

$$
S_{n}(g)=\int_{\Omega} g h_{n} d \mu \text { and } S(g)=\int_{\Omega} g h d \mu
$$

for $g$ in $L_{\infty}\left(\mu, X^{* *}\right)$. Since each $h_{n}$ takes on only a finite number of values, each $S_{n}$ is a compact operator. Moreover, we have that

$$
\left\|\left(S-S_{n}\right)(g)\right\| \leqq \int_{\Omega}\|g\|\left\|h-h_{n}\right\| d \mu \leqq\|g\|_{\infty} \int_{\infty}\left\|h-h_{n}\right\| d \mu
$$


for all $g$ in $L_{\infty}\left(\mu, X^{* *}\right)$. It follows immediately that the operator $S$ is compact. The adjoint of $S$ is the operator $y^{*} \rightarrow y^{*} h(\cdot)$ and hence by Schauder's theorem is also compact. But $y_{n}^{*} h(\cdot)=y_{n}^{*} f(\cdot)$ a.e. This contradicts

$$
\left\|y_{n}^{*} f(\cdot)-y_{m}^{*} f(\cdot)\right\|_{L_{1}\left(\mu, X^{*}\right)}>\delta
$$

for $m \neq n$ and establishes that the set $A$ is compact.

Now choose $y_{n}^{*}$ in $Y^{*}$ such that $y_{n}^{*}(\cdot)$ is dense in $A$. If $h$ is constructed as above for this sequence $\left(y_{n}^{*}\right)$, then $h$ is measurable and so, by Egoroff's theorem, for all $\delta>0$ there is a set $E$ in $\Sigma$ with $\mu(\Omega \backslash E)<\delta$ such that $h \chi_{E}$ can be approximated uniformly by simple functions. Fix $\delta>0$ and choose such a set $E$. It follows that the sequence $y_{n}^{*} f(\cdot) \chi_{E}=y_{n}^{*} h(\cdot) \chi_{E}$ is relatively compact in $L_{\infty}\left(\mu, X^{*}\right)$. Since this sequence is $L_{\infty}\left(\mu, X^{*}\right)$-dense in $\left\{y^{*} f(\cdot)_{\chi_{E}}:\left\|y^{*}\right\| \leqq 1\right\}$, this set is relatively compact in $L_{\infty}\left(\mu, X^{*}\right)$.

Now define $T: Y^{*} \rightarrow L_{\infty}\left(\mu, X^{*}\right)$ by $T y^{*}=y^{*} f(\cdot)_{x_{E}}$. Then $T$ is compact and as an operator on $L_{1}(\mu, X), T^{*}: L_{1}(\mu, X) \rightarrow Y^{* *}$ is compact. Notice that the dominated convergence theorem ensures that $T$ is $w^{*}$ to $w^{*}$ sequentially continuous. Thus, if $y^{* *}$ is in $T^{*}\left(L_{1}(\mu, X)\right)$, then $y^{* *}$ is a weak ${ }^{*}$ sequentially continuous functional on $Y^{*}$. But since $Y$ is weakly compactly generated, this means $y^{* *}$ is a $w^{*}$ continuous functional on $Y^{*}$ [3, p. 148]. Hence, $T^{*}\left(L_{1}(\mu, X)\right)$ is contained in $Y$. Theorem 2 now produces a Bochner integrable $g: E \rightarrow K(X, Y)$ such that

$$
T^{*}(k)=\int_{E} k g d \mu
$$

for all $k$ in $L_{1}(\mu, X)$. But, if $y^{*}$ is in $Y^{*}$, then $T^{* *} y^{*}=y^{*} g$. It follows that $y^{*} g=y^{*} f$ a.e. on $E$. Since $\mu(\Omega \backslash E)<\delta$, this completes the proof.

Theorem 2 does not hold for weakly compact operators. To see this, let $\Omega$ be the unit interval endowed with Lebesgue measure and let $r_{n}(\cdot)$ be the $n$th Rademacher function i.e., $r_{n}(\omega)=\operatorname{signum}\left(\sin 2^{n} \pi \omega\right)$. Consider the function $g:[0,1] \rightarrow L\left(\ell_{2}, \ell_{2}\right)$ defined by $g(\omega)\left(\alpha_{n}\right)=\left(r_{n}(\omega) \alpha_{n}\right)$ for all $\left(\alpha_{n}\right) \in \ell_{2}$. The function $g$ is not essentially separably valued, since if $\omega_{1}$ and $\omega_{2}$ are different numbers in $[0,1]$ there exists a Rademacher function $r_{n}$ with $\left|r_{n}\left(\omega_{1}\right)-r_{n}\left(\omega_{2}\right)\right|=2$ and hence, $\| g\left(\omega_{1}\right)-$ $g\left(\omega_{2}\right) \|_{L\left(\epsilon_{2}, \epsilon_{2}\right)} \geqq 2$. Thus, $g$ is not measurable. Define an operator $T: L_{1}\left(\mu, \ell_{2}\right) \rightarrow \ell_{2}$ by

$$
T(f)=\int_{[0,1]} f g d \mu
$$

and note that $T$ is weakly compact. If $T$ were representable by a kernel, then that kernel would be equal to $g$ a.e. and so $g$ would be 
measurable, which is a contradiction. However, we can use Proposition 4 to obtain a representation theorem for weakly compact operators by imposing further conditions on $X^{*}$.

TheOREm 5. Let $X^{*}$ be a separable Schur space. Then there is an isometric isomorphism between the space of weakly compact operators $W\left(L_{1}(\mu, X), Y\right)$ and the subspace of $L_{\infty}(\mu, W(X, Y))$ consisting of those functions whose essential range is in the uniformly weakly compact operators. In fact, $T$ in $W\left(L_{1}(\mu, X, Y)\right)$ and $g$ in $L_{\infty}(\mu$, $W(X, Y)$ ) are in correspondence if, and only if,

$$
T(f)=\int_{\Omega} f g d \mu
$$

for all $f$ in $L_{1}(\mu, X)$.

Proof. Let $T$ be in $W\left(L_{1}(\mu, X), Y\right)$. By the Factorization Lemma [2, p. 314], there is a reflexive space $R$ and operators $S: L_{1}(\mu, X) \rightarrow$ $R$ and $J: R \rightarrow Y$ such that $T=J S$. Suppose $S$ is representable by a measurable kernel $h: \Omega \rightarrow L(X, R)$. Then $T$ is representable by the measurable kernel $g: \Omega \rightarrow L(X, Y)$ given by $g(\omega) x=J h(\omega) x$ for all $x$ in $X$ and $\omega$ in $\Omega$. Hence, without loss of generality, we may assume that $Y$ is reflexive.

Let $G: \Sigma \rightarrow L(X, Y)$ be the representing measure of $T$ i.e.,

(i) $G(E) x=T\left(x \chi_{E}\right)$ for all $x$ in $X$ and $E$ in $\Sigma$

(ii) $T(f)=\int_{\Omega} f d G$ for all $f$ in $L_{1}(\mu, X)$ and

$$
\|T\|=\sup _{\mu E>0} \frac{\|G(E)\|}{\mu E} .
$$

An appeal to $[10$, p. 345] produces a bounded function $g: \Omega \rightarrow$ $L(X, Y)$ such that

(1) $g(\cdot) x: \Omega \rightarrow Y$ is Bochner integrable for all $x$ in $X$ and

(2) $G(E) x=\int_{E} g(\omega) x d \mu(\omega)$ for all $x$ in $X$ and $E$ in $\Sigma$.

It follows quickly from the density of simple functions in $L_{1}(\mu, X)$ that

$$
T(f)=\int_{\Omega} g f d \mu
$$

for all $f$ in $L_{1}(\mu, X)$. Consider, for each $y^{*}$ in $Y^{*}$, the functions $y^{*} g(\cdot): \Omega \rightarrow X^{*}$. Since these functions are separably valued and weak* measurable, they are measurable by [4, p. 42]. Now $L(X, Y)=$ $K(X, Y)$, since $X^{*}$ is a Schur space and $Y$ is reflexive. Consequently, Proposition 4 now produces a bounded measurable $h: \Omega \rightarrow K(X, Y)$ 
such that, for each $y^{*}$ in $Y^{*}, y^{*} g(\cdot)=y^{*} h(\cdot) \mu$-a.e. Thus, for all $y^{*}$ in $Y^{*}$ and $f$ in $L_{1}(\mu, X)$ we have that

$$
\begin{aligned}
\left\langle y^{*}, T f\right\rangle & =\int_{\Omega}\left\langle y^{*}, g(\omega) f(\omega)\right\rangle d \mu(\omega) \\
& =\int_{\Omega}\left\langle y^{*}, h(\omega) f(\omega)\right\rangle d \mu \\
& =y^{*}\left(\int_{\Omega} h f d \mu\right)
\end{aligned}
$$

and so

$$
T(f)=\int_{\Omega} h f d \mu
$$

It follows easily that

$$
h(\omega) x \leqq \overline{T\left\{f: f \text { in } L_{1}(\mu, X),\|f\|_{1} \leqq 1\right\}}
$$

for almost all $\omega$ in $\Omega$ and all $x$ in $X$ with $\|x\| \leqq 1$. Hence, the essential range of $h$ consists of uniformly weakly compact operators.

The converse is proved in the same way as in Theorem 2 so we omit the proof.

Our final result follows from Theorem 5 in the same way that Corollary 3 follows from Theorem 2 so the proof is omitted.

CoROllary 6. Let $X^{*}$ be a separable Schur space and let $G: \Sigma \rightarrow$ $K(X ; Y)$ be a $\mu$-continuous vector measure of bounded variation. If, for each $E_{1}$ in $\Sigma$ with $\mu E_{1}>0$, there exists an $E_{2}$ in $\Sigma$ with $E_{2} \subseteq E_{1}$ and $\mu\left(E_{2}\right)>0$ such that

$$
\left\{\frac{G(E) x}{\mu E}: x \text { in } X, E \text { in } \Sigma, E \subseteq E_{2}, \mu E>0,\|x\| \leqq 1\right\}
$$

is relatively weakly compact, then there exists a Bochner integrable $g: \Omega \rightarrow K(X, Y)$ such that

$$
G(E)=\int_{E} g d \mu
$$

for each $E$ in $\Sigma$.

ACKNowledgment. The author is grateful to Professor J. J. $\mathrm{Uhl}$, Jr. for his constant advice and encouragement during the preparation of this paper.

\section{REFERENCES}

1. D. Amir and J. Lindenstrauss, The structure of weakly compact subsets in Banach spaces, Ann. of Math., (2) 88 (1968), 35-46. 
2. W. J. Davis, T. Figiel, W. B. Johnson, A Pelczynski, Factoring weakly compact operators, J. Functional Analysis, 17 (1974), 311-327.

3. J. Diestel, Geometry of Banach spaces-Selected Topics, Lecture Notes in Math. v. 485, Springer-Verlag, Berlin and New York, 1975.

4. J. Diestel and J. J. Uhl, Jr., Vector Measures, Math. Surveys No. 15, American Mathematics Society, Providence, 1977.

5. N. Dinculeanu, Vector Measures, Pergamon Press, New York, 1967.

6. N. Dunford and B. J. Pettis, Linear operations on summable functions, Trans. Amer. Math. Soc., 47 (1940), 323-392.

7. N. Dunford and J. T. Schwartz, Linear Operators-Part I, Interscience, New York and London, 1958.

8. N. J. Kalton, Spaces of compact operators, Math. Ann., 208 (1974), 267-278.

9. B. J. Pettis, Differentiation in Banach spaces, Duke Math. J., 5 (1939), 254-269.

10. - On the Radon-Nikodym theorem, Vector Space Measures and Applications I (Proc. Conf. at Dublin, 1977), Lecture Notes 644, Springer-Verlag, Berlin, (1978), 340-355.

11. R. S. Phillips, On linear transformations, Trans. Amer. Math. Soc., 48 (1940), 516541 .

Received September 24, 1979 and in revised form February 15, 1980. This work is a portion of the author's $\mathrm{Ph}$. D. thesis done at The University of Illinois under the direction of Professor J. J. Uhl, Jr.

The Catholic University of America

Washington, DC 20064

Current address: Texas A \& M University

College Station, TX 77843 



\section{PACIFIC JOURNAL OF MATHEMATICS}

\section{EDITORS}

DONALD BABBITT (Managing Editor)

University of California

Los Angeles, CA 90024

HUGo RossI

University of Utah

Salt Lake City, UT 84112

C. C. MOORE and ANDREW OGG

University of California

Berkeley, CA 94720
J. DUGUNDJI

Department of Mathematics

University of Southern California

Los Angeles, CA 90007

R. FinN and J. Milgram

Stanford University

Stanford, CA 94305

\section{ASSOCIATE EDITORS}
R. ARENS
E. F. BECKENBACH
B. H. NeumanN
F. WOLF
K. Yoshida

\section{SUPPORTING INSTITUTIONS}

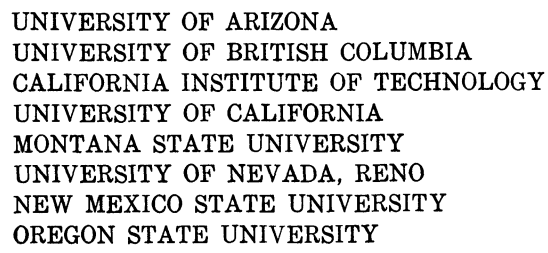

UNIVERSITY OF ARIZONA

UNIVERSITY OF BRITISH COLUMBIA CALIFORNIA INSTITUTE OF TECHNOLOGY

UNIVERSITY OF CALIFORNIA

MONTANA STATE UNIVERSITY

UNIVERSITY OF NEVADA, RENO

NEW MEXICO STATE UNIVERSITY

OREGON STATE UNIVERSITY

\author{
UNIVERSITY OF OREGON \\ UNIVERSITY OF SOUTHERN CALIFORNIA \\ STANFORD UNIVERSITY \\ UNIVERSITY OF HAWAII \\ UNIVERSITY OF TOKYO \\ UNIVERSITY OF UTAH \\ WASHINGTON STATE UNIVERSITY \\ UNIVERSITY OF WASHINGTON
}

The Supporting Institutions listed above contribute to the cost of publication of this Journal, but they are not owners or publishers and have no responsibility for its content or policies.

Mathematical papers intended for publication in the Pacific Journal of Mathematics should be in typed form or offset-reproduced, (not dittoed), double spaced with large margins. Please do not use built up fractions in the text of the manuscript. However, you may use them in the displayed equations. Underline Greek letters in red, German in green, and script in blue. The first paragraph or two must be capable of being used separately as a synopsis of the entire paper. Please propose a heading for the odd numbered pages of less than 35 characters. Manuscripts, in triplicate, may be sent to any one of the editors. Please classify according to the scheme of Math. Reviews, Index to Vol. 39. Supply name and address of author to whom proofs should be sent. All other communications should be addressed to the managing editor, or Elaine Barth, University of California, Los Angeles, California, 90024.

50 reprints to each author are provided free for each article, only if page charges have been substantially paid. Additional copies may be obtained at cost in multiples of 50 .

The Pacific Journal of Mathematics is issued monthly as of January 1966. Regular subscription rate: $\$ 102.00$ a year (6 Vols., 12 issues). Special rate: $\$ 51.00$ a year to individual members of supporting institutions.

Subscriptions, orders for numbers issued in the last three calendar years, and changes of address shoud be sent to Pacific Journal of Mathematics, P.O. Box 969, Carmel Valley, CA 93924, U.S.A Old back numbers obtainable from Kraus Periodicals Co., Route 100, Millwood, NY 10546.

\section{PUBLISHED BY PACIFIC JOURNAL OF MATHEMATICS, A NON-PROFIT CORPORATION}

Printed at Kokusai Bunken Insatsusha (International Academic Printing Co., Ltd.). 8-8, 3-chome, Takadanobaba, Shinjuku-ku, Tokyo 160, Japan. 


\section{Pacific Journal of Mathematics}

Vol. 92, No. $2 \quad$ February, 1981

Bruce Allem Anderson and Philip A. Leonard, Sequencings and Howell designs

Kevin T. Andrews, Representation of compact and weakly compact

operators on the space of Bochner integrable functions . . . . . . . . 257

James Glenn Brookshear, On the structure of hyper-real $z$-ultrafilters . . . . . 269

Frank John Forelli, Jr., A necessary condition on the extreme points of a class of holomorphic functions. II ...................... 277

Richard J. Friedlander, Basil Gordon and Peter Tannenbaum, Partitions of groups and complete mappings ......................... 283

Emden Robert Gansner, Matrix correspondences of plane partitions ......295

David Andrew Gay and William Yslas Vélez, The torsion group of a radical extension ..........................................

André (Piotrowsky) De Korvin and C. E. Roberts, Convergence theorems for some scalar valued integrals when the measure is Nemytskii ...... 329

Takaŝi Kusano and Manabu Naito, Oscillation criteria for general linear ordinary differential equations $\ldots \ldots \ldots \ldots \ldots \ldots \ldots \ldots \ldots \ldots \ldots \ldots \ldots \ldots \ldots \ldots$

Vo Thanh Liem, Homotopy dimension of some orbit spaces .......... 357

Mark Mahowald, $b o$-resolutions . . . . . . . . . . . . . . . . . . . 365

Jan van Mill and Marcel Lodewijk Johanna van de Vel, Subbases, convex

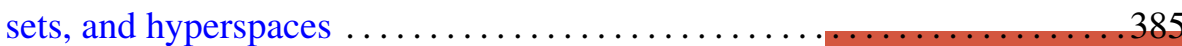

John F. Morrison, Approximations to real algebraic numbers by algebraic numbers of smaller degree $\ldots \ldots \ldots \ldots \ldots \ldots \ldots \ldots \ldots \ldots \ldots \ldots \ldots$

Caroline Series, An application of groupoid cohomology . . . . . . . . . . 415

Peter Frederick Stiller, Monodromy and invariants of elliptic surfaces . . . 433 Akihito Uchiyama, The factorization of $H^{p}$ on the space of homogeneous



Warren James Wong, Maps on simple algebras preserving zero products.

II. Lie algebras of linear type 